\title{
Détermination de la durée de vie géochimique du stock de déchets miniers de Carnoulès (France) à partir d'un modèle hydrodynamique.
}

\author{
Kouadio KofFI ${ }^{1 *}$, Inza DoumouYA ${ }^{1}$, Bi Tié Albert GoulA ${ }^{1} \&$ Séverin PISTRE ${ }^{2}$ \\ ${ }^{1}$ Laboratoire Géosciences et Environnement, UFR Sciences et Gestion de l'Environnement, Université d'Abobo-Adjamé, 02 BP 802 \\ Abidjan 02 (Côte d'Ivoire). \\ ${ }^{2}$ Laboratoire d'hydrosciences - Maison des Sciences de l'eau, Université de Montpellier II, 300 Avenue Emile Jambreau 34090 Montpellier. \\ *Auteur pour les correspondances (E-mail: kouadiok1@yahoo.fr) \\ Reçu le 23-02-2006, accepté le 23-01-2007.
}

\section{Résumé}

Le stock de déchets miniers de Carnoulès forme un aquifère bicouche constitué de sable très fin à sulfure et de sable fin à passage de blocs grossiers. L'écoulement de l'eau à l'intérieur est guidé par un drain souterrain. Le modèle hydrodynamique a permis d'évaluer les flux de matières à partir de la reconstitution de débits à l'exutoire. Les fortes teneurs en sulfates de l'eau observées à la source proviennent de la dissolution de la pyrite qui détermine l'essentiel des réactions géochimiques à l'intérieur du stock de déchets miniers. La durée de vie du stock de déchets miniers estimée à partir des flux annuels de pyrite oxydée est de plus de 100 ans. Les méthodes de rémédiation appropriées ne doivent donc pas être des installations qui exigeront un entretien continu compte tenue de cette longue durée de vie.

Mots clés : Drainage acide minier, modélisation, hydrodynamique, Modflow

\section{Abstract}

Geochemical lifetime determination of the mining waste stock of Carnoulès (France) by a hydrodynamic model.

The mining waste stock of Carnoulès is a double-layered aquifer made up of very fine clay and sand with sulphide and of sand ends to passage of coarse blocks. The groundwater circulation is guided by an underground drain. The hydrodynamic model made it possible to evaluate matter flows starting from the reconstitution of flows to the catchment discharge system. It arises a strong content sulphate of water coming from the dissolution of the pyrite which guides the main geochemical reactions inside the stock of mining waste. The lifetime considered starting from annual oxidized pyrite flows is of more than 100 years. Methods of remediation should not be installations requiring a continuous maintenance.

Key words: Mining acid drainage, modelling, hydrodynamics, Modflow

\section{Introduction}

Les déchets miniers constituent un problème environnemental majeur dans le monde à cause des effets néfastes produits sur l'écosystème en général. Certains déchets contiennent des composés sulfurés (la pyrite) qui, au contact de l'eau de pluie chargée en oxygène, génèrent des eaux acides riches en métaux lourds toxiques (Marée et al., 2004). Ce processus est connu sous le nom de drainage acide minier. L'acidité résulte de réactions chimiques catalysées par les bactéries (Nordstrom, 1982). Ces eaux acides peuvent contaminer les ressources en 
eau environnantes, la flore et la faune. C'est le cas du stock de déchets de l'exploitation de Plomb et Zinc de Carnoulès (France). Le pH de ces eaux est de 3 avec une concentration en arsenic supérieure à 300 mg/L (Leblanc et al., 2001 ; Casiot et al., 2001, 2003 ; Morin et al., 2003).

Afin de circonscrire cette pollution, un aménagement du stock de déchets miniers a été réalisé après la fermeture de la mine en 1962. Ces déchets ont été confinés dans un bassin versant avec drainage des eaux d'infiltration. A la sortie du bassin versant, une digue en béton maintient tout le stock de déchets miniers. Au pied de cette digue, un canal a été creusé pour recueillir les lixiviats afin d'éviter la pollution diffuse. Cependant, des fissures sont apparues dans la digue, ce qui rend difficile la canalisation de l'eau et difficile l'évaluation des flux de polluants dans une perspective de rémédiation.

L'objectif de cette étude est de déterminer la durée de vie géochimique du stock de déchets miniers dans la perspective de la mise place d'une méthode de rémédiation appropriée. Pour atteindre cet objectif, les débits d'eau à la sortie du stock de déchets miniers ont été reconstitués à partir d'un modèle hydrodynamique. Sur la base de ces débits reconstitués, des flux annuels de solutés sont estimés. La durée de vie géochimique du stock de déchets est supposé être le temps au bout duquel toute l'arsenopyrite contenue est oxydée.

\section{Matériels et méthodes}

\subsection{Description du site}

Le site minier de Carnoulès est situé au Sud de la France dans la commune de Saint Sébastien d'Aigrefeuille (Gard) (Fig.1). Ce site appartient au bassin versant hydrologique d'Anduze est marqué par un cours d'eau principal qui est le Gardon. Ses principaux affluents sont le Gardon Saint Jean, le Gardon Mialet et l'Amous (fig.1). Le régime hydrographique est fortement influencé par le relief à fortes pentes qui favorisent l'inondation des vallées et l'expansion de la pollution minière pendant les épisodes pluvieux.

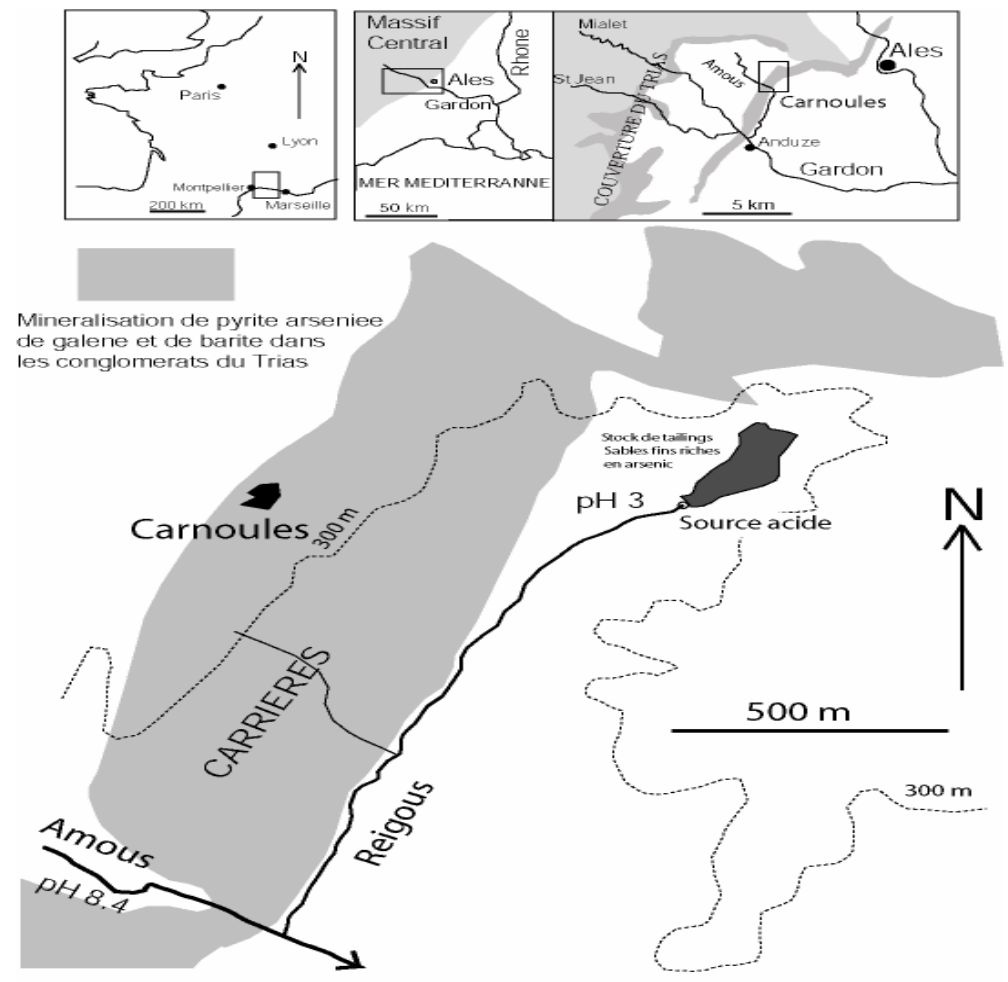

Figure 1 : Situation géographique du site minier de Carnoulès. 
Au plan géologique, trois domaines géologiques majeurs se dégagent (Alkaaby, 1986) (Fig. 2). Le socle hercynien, la couverture mésozoïque de la bordure cévenole contrôlée par des paléo-reliefs du socle et le bassin du crétacé avec principalement des roches dolomitiques (Viallette \& Sabourdy, 1977 ; Leenhart, 1972). La région est contrôlée par des structures faillées tardi-hercyniennes (Seguret \& Proust, 1965). Elles ont favorisé une forte minéralisation dans une tranche lithologique conglomératique. Au total 55 à 65.000 tonnes de Plomb, 35.000 tonnes de Zinc et 85 à 100 tonnes d'Argent ont été exploités (Arthaud \& Matte, 1975 ; Alkaaby, 1986).

Ces exploitations ont laissé $1 \mathrm{~km}^{2}$ de carrières et des millions de tonnes de sables fins à sulfures avec 5 à $10 \%$ de pyrite rejetés dans un vallon lors du traitement du minerai. Ce vallon est le lit du ruisseau Reigous qui alimente la rivière l'Amous.

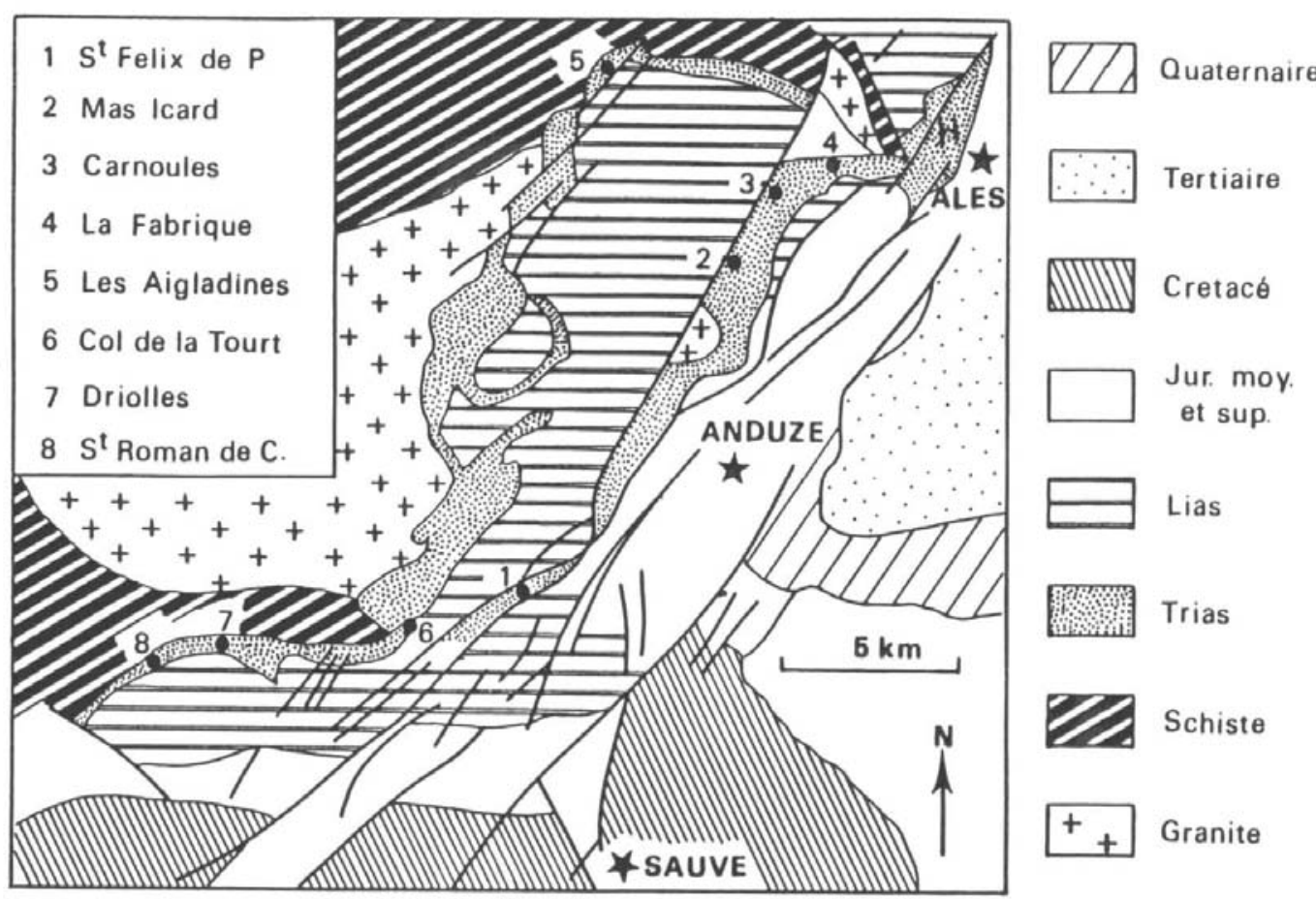

Figure 2 : Schéma géologique de la région étudiée (Alkaaby, 1986).

\subsection{Modélisation hydrodynamique et analyse hydrogéochimique}

\subsubsection{Equipements et mesures}

Cinq sondages à petits diamètres et 11 forages (vp1, vp2, vp4 forés en 1982 ; s1, s3, s5, s7, s10 forés en 1998, s13, s14 forés en 2000 et s12 forés en 2001) ont servi à la reconstitution du mûr de l'aquifère et à la surveillance du niveau de l'eau (Fig. 5). Quatre de ces forages ont été équipés de capteurs de pressions de types DL-
$\mathrm{N}$ et SCAINE (précision de $+/$ - 0,1\% E.M) pour mesurer le niveau de l'eau en continue. Le pas de temps de mesure est fixé à 30 minutes. Ces mesures du niveau d'eau servent de points d'observation pendant le calage du modèle en régime permanent et transitoire. Les erreurs sur ces mesures fournies par les capteurs de pressions sont corrigées par des mesures ponctuelles dans tous les forages au moins deux fois par mois. Un débitmètre électromagnétique est installé pour mesurer les débits de l'eau en continue. 
Le site est également équipé d'un pluviomètre à auget pour mesurer la quantité d'eau qui tombe. La quantité d'eau qui s'infiltre a été estimée à partir de l'évapotranspiration estimée par la méthode de Thornwhaite.

La détermination des paramètres hydrodynamiques des unités lihostratigraphiques identifiées à partir des sondages s'est faite par des méthodes d'essai de pompage, de slug test, d'analyse granulométrique et d'infiltrabilité à double anneau pour les couches superficielles.

Le modèle hydrodynamique utilisé est le modèle MODFLOW de US Geology Survey (USGS). La version appliquée est un module du code Groundwater Modelling System (GMS) du laboratoire EMRL (Environment Modeling Research Laboratory) de l'Université de Young Brigham (Zheng, 1990). Ce modèle a été appliqué avec succès par Winston (1998), Zhen (1990) ; Hill (1994) et Konikow et al. (1996).

\subsubsection{Condition d'application du modèle}

Le stock de déchets n'échange pas avec d'autres systèmes. La recharge en eau se fait donc uniquement par la pluie diminuée de l'évapotranspiration. Des flux nuls sont appliqués à l'ensemble des contours du stock de déchets minier sauf à la sortie où une charge est imposée. Pour une précision sur les interpolations des niveaux d'eau modélisés, un maillage rectangulaire de $20 \mathrm{~m}$ sur $10 \mathrm{~m}$ est choisi.

En fonction des paramètres hydrodynamiques estimés, deux couches sont considérées; une première couche superficielle isotrope et homogène constituée de l'argile de couverture et de sables fins superficiels et une deuxième couche anisotropique et hétérogène. Cette deuxième couche constituée de sables grossiers avec parfois des passages de blocs grossiers a été divisée en quatre parties en fonction des conductivités hydrauliques estimées (Fig. 3). La recharge choisie est la valeur moyenne des recharges de décembre 2000 à avril 2001. Pendant cette période, les niveaux de l'eau dans le système varient peu. On fait l'hypothèse selon laquelle le système fonctionne en régime pseudo-permanent et la valeur moyenne de la recharge sur cette période est considérée constante.

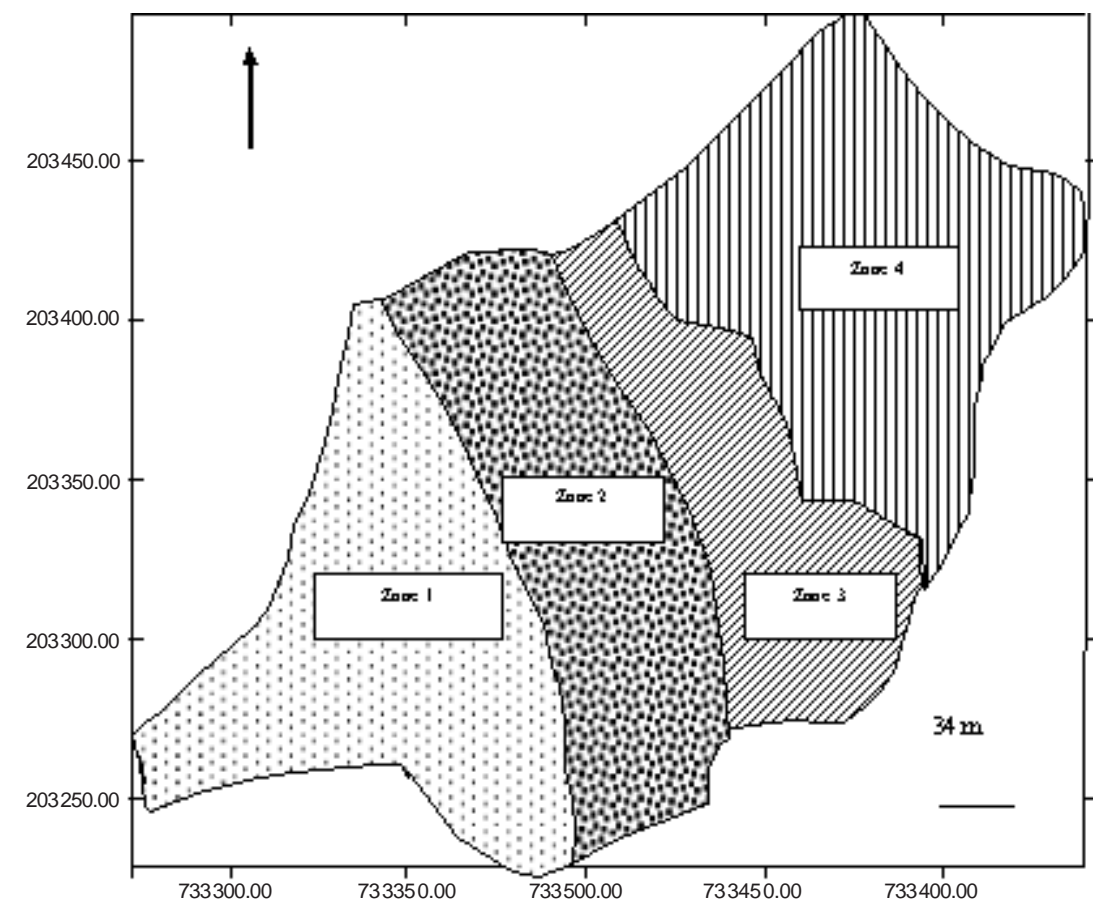

Figure 3 : Zones de conductivités hydrauliques homogènes choisies dans la couche 2 avant le calage en régime permanent. 


\subsubsection{Analyses chimiques}

Au pied de la digue, les sulfates, le plomb et le zinc ont été analysés dans le lixiviat. En effet, ces éléments sont les principaux éléments chimiques de la phase solide du stock de déchets. Les prélèvements de lixiviat ont été effectués au moins une fois par mois sur la période de 2000 à 2002. Les échantillons sont prélevés dans des flacons en polyéthylène et filtrés sur des membranes millipores $0,45 \mathrm{~mm}$. Ceux qui sont destinés à l'analyse des métaux sont acidifiés avec l'acide nitrique ultra pure à $60 \%$. Les analyses chimiques de ces éléments ont été effectuées par ICP-AES. Les protocoles d'analyse sont décrits par ElbazPoulichet et al., (1999) et Bozau et al., (2004).

\subsubsection{Calcul des flux de matières et estimation de la durée de vie}

Le processus de minéralisation dans le stock de déchets miniers de Carnoulès libère essentiellement des sulfures (pyrite, galène et un peu de sphalérite) dans une gangue de quartz, orthose, barytine et micas altérés (Alkaaby et al, 1985). Les proportions en masse des composés sulfurés sont 5 à $10 \%$ de pyrite arséniée, $1 \%$ de galène et des traces de sphalérite. L'oxydation de ces sulfures libère des ions sulfates et des métaux suivant les équations :

$\mathrm{FeS}_{2}(\mathrm{~s})+7 / 2 \mathrm{O}_{2}+\mathrm{H}_{2} \mathrm{O} \longrightarrow \mathrm{Fe}^{2+}+2 \mathrm{SO}_{4}^{2-}+2 \mathrm{H}^{+}$

(Pyrite)

$\mathrm{PbS}(\mathrm{s})+2 \mathrm{O}_{2}(\mathrm{aq}) \longrightarrow \mathrm{Pb}^{2+}+\mathrm{SO}_{4}^{2-}$

(Galène)

$\mathrm{ZnS}(\mathrm{s})+2 \mathrm{O}_{2}(\mathrm{aq}) \longrightarrow \mathrm{Zn}^{2+}+\mathrm{SO}_{4}^{2-}$

(Sphalérite)

D'autres sources de sulfates telle que la barytine peuvent être considérées, mais elles restent négligeables à cause de la faible dissolution de la barytine dans les eaux de drainage acides de Carnoulès (Leblanc, 2002).

D'une façon générale, on peut supposer que la quantité de sulfates présente $\left|S O_{4}^{2-}\right|$ à la sortie du système acide est la somme des quantités de sulfates générées simultanément par l'oxydation de la pyrite $\left[\text { so }_{\text {?- }}^{2-}\right]_{x_{\mathrm{er}} 2}$ d'une part, et d'autre part par des réactions de dissolution de la galène $\left[\mathrm{SO}^{3-}\right]_{\mathrm{PLS}}$ et de la sphalérite $\quad\left[\mathrm{SO}_{4}^{2-}\right]_{\mathrm{Zn} S}$. Soit

$\left[\mathrm{SO}_{4}^{2-}\right]_{T}=\left[\mathrm{SO}_{4}^{2-}\right]_{P b S}+\left[\mathrm{SO}_{4}^{2-}\right]_{\mathrm{ZnS}}+2\left[\mathrm{SO}_{4}^{2-}\right]_{\mathrm{FeS} 2}(4)$

Cette équation équivaut aussi à :

$\left[\mathrm{SO}_{4}^{2-}\right]_{T}=[\mathrm{Pb}]_{\mathrm{PbS}}+[\mathrm{Zn}]_{\mathrm{ZnS}}+2\left[\mathrm{SO}_{4}^{2-}\right]_{\mathrm{FeS} 2}$

avec $[\mathrm{Pb}]_{P b S}$ et, $[\mathrm{Zn}]_{\mathrm{ZnS}}$ étant respectivement les flux de plomb et de zinc à la sortie du système. La stœchiométrie du fer étant assez complexe dans un milieu aussi réactif, la quantité de sulfate produite par l'oxydation de la pyrite est déterminée par :

$2\left[\mathrm{SO}_{4}^{2-}\right]_{\mathrm{FeS} 2}=\left[\mathrm{SO}_{4}^{2-}\right]_{T}-\left[[\mathrm{Zn}]_{\mathrm{ZnS}}+[\mathrm{Pb}]_{\mathrm{PbS}}\right]$

Les flux $\left(\left(F_{g}\right)\right.$ en mole/s) de matières pour un éléments chimique $g$ ( $g=\{$ sulfate, plomb, zinc $\}$ ) sont donnés par l'équation suivante :

où

$$
F_{g}=\frac{Q C}{\mu}
$$

$Q \quad\left(L^{-1}{ }^{-1}\right)$ : débit moyen mensuel modélisé ;

$C$ en $\left(\right.$ g. $\left.\mathrm{L}^{-1}\right)$ : les concentrations moyennes mensuelles ;

$\mu$ (g.mole $\left.{ }^{-1}\right)$ : la masse molaire.

La connaissance des flux de sulfates totaux, de zinc et de plomb à la sortie du système permet d'accéder aux flux de sulfate provenant de l'oxydation de la pyrite.

La durée de vie $T$ du système a été estimée à partir de l'équation suivante :

$$
T=\frac{2 Q_{\text {gir }}}{Q_{\text {uw }}}
$$

où $T$ : la durée de vie du système en années, 
$Q_{\text {pyr }}$ : la quantité totale de pyrite en moles disponible,

$Q_{\text {sulf: }}$ la quantité annuelle de sulfate en moles produite,

$Q_{p y r}$ : est déterminée à partir du volume du stock de déchets miniers.

Le volume total du stock de déchets miniers est estimé à partir de la surface donnée par le logiciel GMS (Groundwater Modelling System) et des épaisseurs interpolées à partir des profondeurs de forage. La quantité totale de pyrite à oxyder est calculée à partir de la densité de la phase solide du dépôt déterminée et du pourcentage massique de pyrite contenu dans le stock de déchets miniers.

\section{Résultats et discussions}

\subsection{Modélisation hydrodynamique}

En régime permanent, plusieurs simulations ont été effectuées en considérant différentes valeurs de conductivités hydrauliques et de conductance du drain qui correspondent au mieux à l'état permanent du système. C'est-à-dire celles pour lesquelles les valeurs de charges simulées sont proches de celles observées. Ces valeurs calées sont comparées à celles estimées et sont présentées dans le tableau 1. Les piézométries modélisées et celles mesurées sont présentées dans la Fig. 4 et la Fig. 5.

Tableau 1 : Tableau de comparaison entre les conductivités hydrauliques et la conductance de drains mesurées et celles obtenues après calage $\left(\mathrm{K}_{\mathrm{h}}\right.$ conductivité hydraulique horizontale, $\mathrm{K}_{\mathrm{v}}$ conductivité hydraulique verticale)

\begin{tabular}{|c|c|c|c|c|c|}
\hline & & \multicolumn{2}{|c|}{ Conductivités hydrauliques estimées } & \multicolumn{2}{|c|}{ Conductivités hydrauliques cal'és } \\
\hline \multicolumn{2}{|c|}{$\begin{array}{c}\text { Conductivité } \\
\text { hydraulique Couche } 1\end{array}$} & \multicolumn{2}{|c|}{$3.10^{-4} \cdot 1.4 .10^{-7} \mathrm{~m} / \mathrm{s}$} & \multicolumn{2}{|c|}{$1.10^{7} \mathrm{~m} / \mathrm{s}$ sotrope et homogène } \\
\hline $\begin{array}{c}\text { Conductivité } \\
\text { hydraulique } \\
\text { Couche } 2\end{array}$ & $\begin{array}{l}\text { Zone } 1 \\
\text { Zone } 2 \\
\text { Zone } 3 \\
\text { Zone } 4 \\
\text { Zone } 5 \\
\end{array}$ & $\begin{array}{l}K_{n}=1.10^{-6} \\
K_{n}=9.10^{-9} \\
K_{n}=3.10^{-4} \\
K_{n}=1.10^{-4} \\
K_{n}=3.10^{-4}\end{array}$ & $\begin{array}{l}K_{2}=1210^{-7} \\
K_{2}=3.710^{7} \\
K_{2}=3.10^{7} \\
K_{2}=1.10^{-7} \\
K_{2}=4.510^{-4}\end{array}$ & $\begin{array}{l}K_{n}=1.10^{-8} \\
K_{n}=2.510^{-6} \\
K_{n}=3 \cdot 10^{-6} \\
K_{n}=9 \cdot 10^{-4} \\
K_{n}=6.3 \cdot 10^{-6}\end{array}$ & $\begin{array}{l}K_{2}=4.10^{\circ} \\
K_{2}=2.4 .10^{7} \\
K_{2}=4.210^{-7} \\
K_{2}=9.10^{-7} \\
K_{2}=6.10^{-4}\end{array}$ \\
\hline \multicolumn{2}{|c|}{$\begin{array}{c}\text { Conductance drain } \\
\text { souterrain }\end{array}$} & & $3.510^{4} \mathrm{~m}^{2} / \mathrm{s}$ & \multicolumn{2}{|c|}{$1.510^{-1} \mathrm{~m}^{2} k$} \\
\hline
\end{tabular}

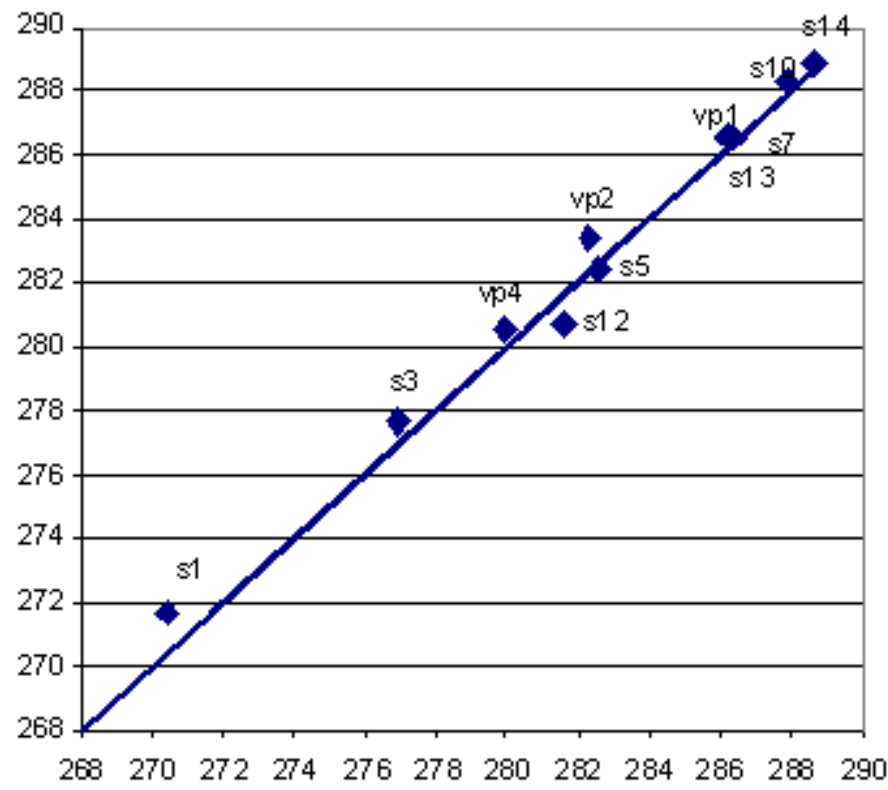

Miveau modélisé

Figure 4 : Comparaison des niveaux d'eaux mesurés et modélisés 


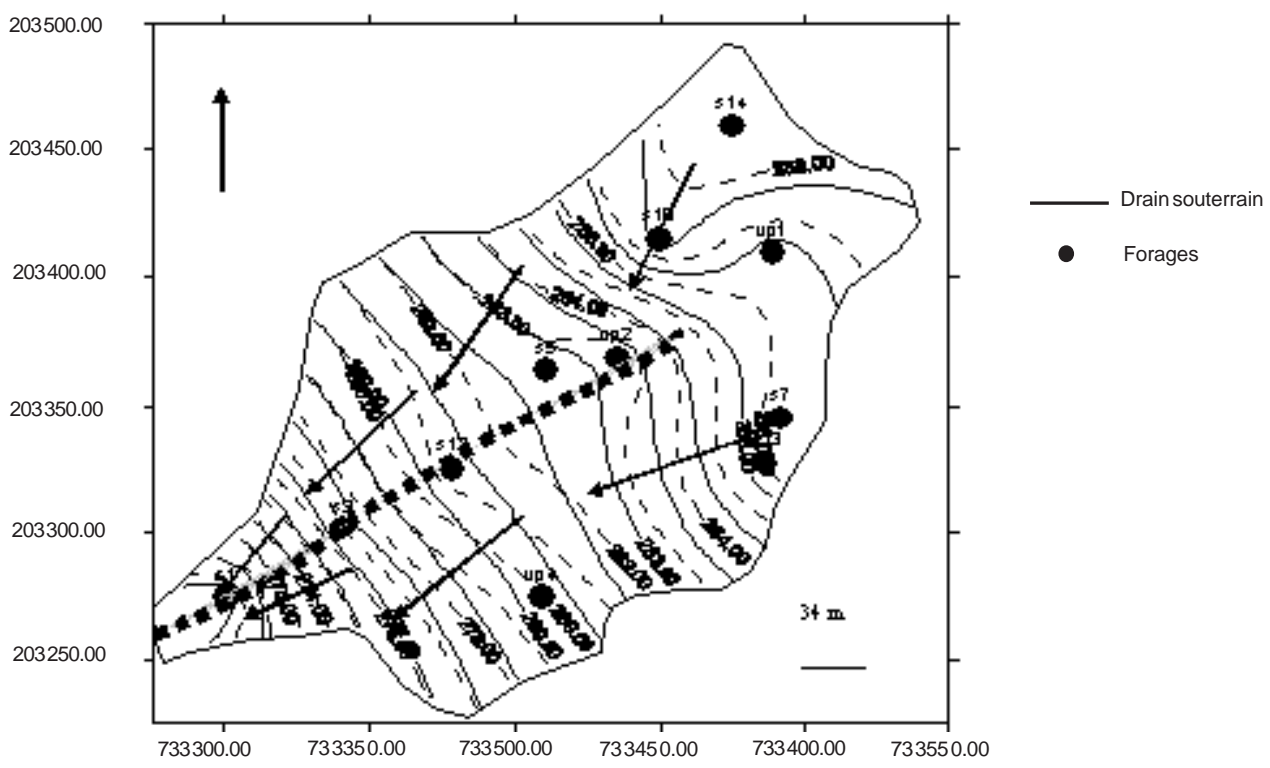

Figure 5 : Comparaison de la répartition spatiale de la piézométrie modélisée et celle mesurée

Les conductivités hydrauliques calées sont dans la gamme de celles estimées par les différentes méthodes. Par ailleurs, sur l'échelle de classification des valeurs de conductivités hydrauliques en fonction des structures lithologiques définie par (Domenico \& Schwartz, 1990), les valeurs estimées correspondent à du sable argileux pour la première couche et à du sable grossier pour la deuxième couche. Ce sont les deux principales couches rencontrées lors des différents sondages. D'ailleurs, la comparaison entre les valeurs de charges générées par le modèle et celles mesurées sur le terrain dans les 15 forages, montre une corrélation acceptable $\left(r^{2}=0.99\right)$ autour de l'axe $x=y$. Au regard de la qualité des écarts produits $(<0,8 \mathrm{~m})$ et de la valeur du débit simulé à la sortie (proche du débit moyen mesuré), ce scénario traduit l'état permanent du fonctionnement de l'aquifère.

L'étude de sensibilité par rapport à la recharge effectuée a montré que la valeur de recharge estimée à partir de l'évapotranspiration par la méthode de Thornthwaite pourrait être considérée comme une surestimation de la recharge; la couverture argileuse du stock de déchets miniers pouvant réduire les infiltrations. Mais, d'autres scénarios étudiés par Koffi et al., (2003) et Bruneel et al., (2005) basés sur des variations physico-chimiques et biologiques de la qualité de l'eau dans le système ont permis de comprendre que cette surestimation est compensée par des apports diffus d'eau souterraine. En effet, il a été prouvé que les déchets miniers de Carnoulès ont été stockés dans une dépression à la limite d'une faille ouverte (Koffi et al., 2003). Ces apports souterrains d'eau par la faille sont certainement irréguliers et donc difficiles à estimer dans un schéma transitoire. De manière indirecte, ils sont pris en compte par une surestimation de la recharge en considérant la valeur de recharge estimée par la méthode de Thornthwaite.

\subsection{Régime transitoire}

En régime transitoire, les conductivités hydrauliques, la conductance du drain et la recharge connues, l'emmagasinement est calé et les débits à la sortie du stock de déchets miniers sont observés. Ces valeurs de débits sont comparées à des valeurs ponctuelles mesurées à la sortie de l'eau (Fig. 6). 


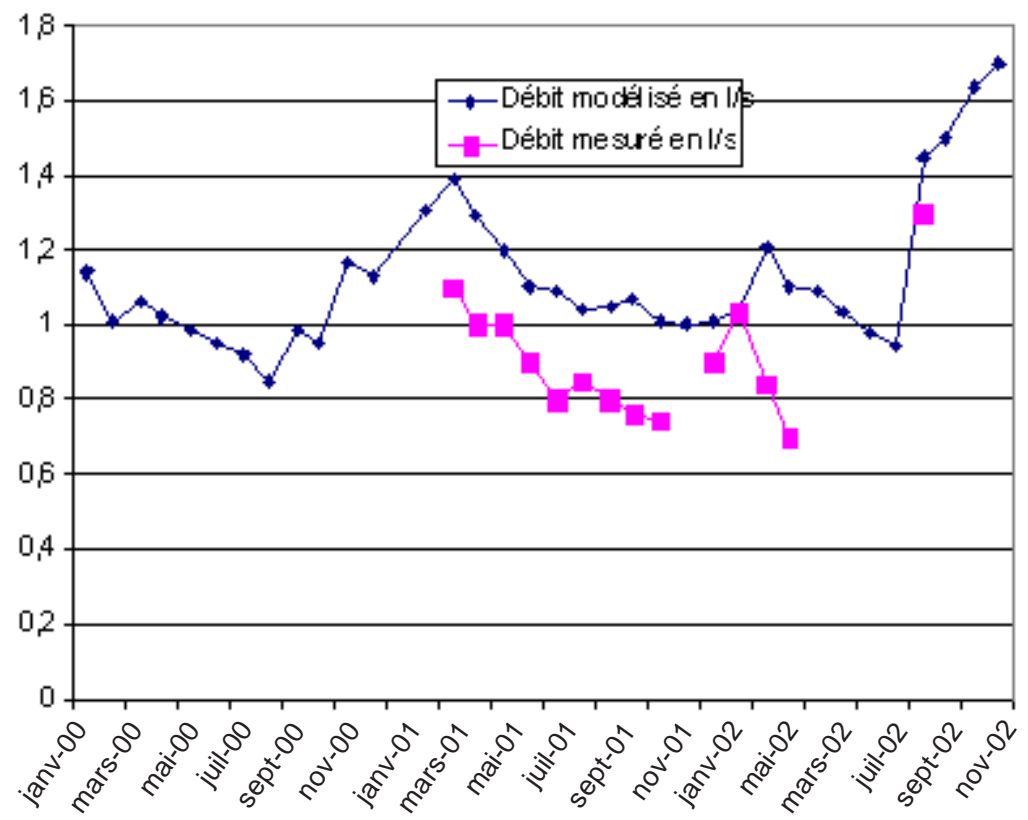

Figure 6 : comparaison entre les débits mensuels modélisés et les débits mesurés.

Les débits modélisés varient de $0,8 \mathrm{~L} . \mathrm{S}^{-1}$ à 1,7 L.S ${ }^{-1}$ sur les trois années d'étude. De façon générale, les valeurs de débits modélisés sont supérieures à celles des débits mesurés avec un écart maximal de 0,5 L.s. ${ }^{-1}$. Aussi, existe-t-il une discontinuité dans les débits mesurés. La discontinuité dans les mesures de débits est liée à un dysfonctionnement du débitmètre soumis aux sables fins polarisés pendant certaines périodes de l'année. En effet, le débit a été mesuré à partir de la tension induite créée par l'eau pendant son passage dans un champ magnétique à l'intérieur du débitmètre. Or, les sables fins qui sortent en grande quantité avec l'eau pendant les périodes pluvieuses modifient considérablement la tension induite et donc les débits mesurés. Ces débits n'ont donc pas été pris en compte.

Les valeurs de débits modélisés sont toujours supérieures à celles des débits mesurés. Cet écart entre les débits modélisés et observés est dû aux pertes d'eau par suintement qui échappent à la mesure. En effet, la digue censée retenir l'eau présente des fissures qui laissent échapper l'eau de façon diffuse pendant une bonne partie de l'année. Cette fuite d'eau est difficile à mesurer avec précision.

Les débits reconstitués par le modèle permettent d'évaluer des flux de matières et d'estimer la durée de vie du système. Ces chroniques de débits mensuels restitués ainsi que les concentrations moyennes mensuelles de $\mathrm{Zn}, \mathrm{Pb}$, $\mathrm{Cu}$ obtenues dans les eaux à la sortie ont permis de calculer les flux mensuels de ces éléments chimiques (Tableau 2).

Les flux mensuels à la source varient de 41 à 172 kmoles.mois ${ }^{-1}$ pour $\mathrm{SO}_{4}{ }^{2-}$, de 8 à 36 kmoles.mois ${ }^{1}$ pour $\mathrm{Pb}$ et de 0,70 à 2,48 kmoles. mois $^{-1}$ pour $\mathrm{Zn}$. Les flux de sulfates totaux sont environ 1000 fois plus importants que la somme des flux de $\mathrm{Pb}$ et de $\mathrm{Zn}$. L'essentiel des sulfates provient donc de l'oxydation de la pyrite.

Les durées de vie géochimiques du stock de déchets miniers calculées sont présentées dans le tableau 3. Elles varient de 128 à 376 ans. La méthode de calcul de la durée de vie, bien que ne tenant pas compte des cinétiques a été utilisée avec succès par Younger et al. (2002) dans d'autres sites miniers. Les déchets miniers de Carnoulès pollueront donc l'environnement sur toute cette période. 
Tableau 2 : Estimation du flux de sulfate (en moles) provenant de la pyrite à la source acide.

\begin{tabular}{|c|c|c|c|c|}
\hline \multirow[b]{2}{*}{ Date } & \multicolumn{4}{|c|}{ Ruxd'e matiènes en moles mois } \\
\hline & $\begin{array}{l}\text { Rux de sufate totaux } \\
\text { estimé à la source }\end{array}$ & Flux de plomb & Fux de zinc & $\begin{array}{c}\text { Ruxde suffate proverart } \\
\text { de la pyrite }\end{array}$ \\
\hline janve0 00 & 141588 & 23 & 1186 & 140378 \\
\hline fér-00 & 125442 & 21 & 1051 & 124370 \\
\hline $\operatorname{mas} 00$ & 131652 & 22 & 1103 & 130527 \\
\hline aur 000 & 126684 & 21 & 1062 & 125602 \\
\hline maio0 & 140333 & 20 & 1030 & 139282 \\
\hline juin-00 & 134663 & 26 & 1186 & 133451 \\
\hline juit-00 & 130410 & 25 & 1148 & 129236 \\
\hline aoû́-00 & 120488 & 23 & 1061 & 119400 \\
\hline sept 00 & 140333 & 27 & 1236 & 139070 \\
\hline odt- 0 & 82773 & 26 & 1186 & 81561 \\
\hline $\begin{array}{l}\text { nove00 } \\
\text { déc00 }\end{array}$ & $\begin{array}{l}101941 \\
98456\end{array}$ & $\begin{array}{l}21 \\
21\end{array}$ & $\begin{array}{l}732 \\
707\end{array}$ & $\begin{array}{c}101187 \\
97728\end{array}$ \\
\hline janve 01 & 122852 & 31 & 1012 & 121809 \\
\hline férs-01 & 114139 & 28 & 940 & 113170 \\
\hline $\operatorname{mars} 01$ & 121109 & 30 & 998 & 120081 \\
\hline$a x=01$ & 172896 & 28 & 926 & 171942 \\
\hline mai-01 & 160834 & 27 & 2490 & 158316 \\
\hline juin-01 & 147431 & 25 & 283 & 145123 \\
\hline juit-01 & 146091 & 25 & 262 & 143804 \\
\hline aoû́-01 & 139389 & 24 & 2158 & 137207 \\
\hline sept 01 & 140729 & 24 & 2179 & 138526 \\
\hline odt-01 & 143410 & 25 & 220 & 141165 \\
\hline nove01 & 135368 & 23 & 2096 & 133249 \\
\hline déo01 & 134028 & 23 & 2075 & 131930 \\
\hline janv-02 & 92882 & 8 & 451 & 92422 \\
\hline fér-02 & 95640 & 8 & 464 & 95168 \\
\hline $\mathrm{mars} 02$ & 111274 & 10 & 540 & 110724 \\
\hline$a v-02$ & 101158 & 9 & 491 & 100658 \\
\hline $\mathrm{mar} 02$ & 71603 & 17 & 708 & 70878 \\
\hline juin-02 & 67662 & 16 & 669 & 66976 \\
\hline juit 02 & 64377 & 15 & 637 & 63725 \\
\hline aô̂́-02 & 61750 & 15 & 611 & 61124 \\
\hline sept 012 & 40129 & 31 & 1010 & $390 \%$ \\
\hline odt -02 & 41513 & 32 & 1044 & 40436 \\
\hline nove 02 & 45387 & 35 & 1142 & 44210 \\
\hline déc 02 & 47048 & 36 & 1184 & 45828 \\
\hline
\end{tabular}

Tableau 3 : Durées de vie du système pour différents scénarios sur la teneur en pyrite du stock de déchets miniers (5 à $10 \%)$

\begin{tabular}{lccc}
\hline Année de référence du flux & 2000 & 2001 & 2002 \\
\hline$Q_{\text {sur }}$ en moles/an & $1,5.10^{6}$ & $1,7.10^{6}$ & $0,8.10^{6}$ \\
$T(5 \%)$ de pyrite & $\mathbf{1 3 5}$ & $\mathbf{1 2 8}$ & $\mathbf{1 8 a}$ \\
$T(7.5 \%)$ de pyrite & $\mathbf{2 0 3}$ & $\mathbf{1 9 2}$ & $\mathbf{2 8 2}$ \\
$T(10 \%)$ de pyrite & $\mathbf{2 7 1}$ & $\mathbf{2 5 5}$ & $\mathbf{3 7 6}$ \\
\hline
\end{tabular}

$T(X \%)$ correspond a la duree de vie du systeme calcule sur la base qu'll exIste X\% de d'arsenopyrite dans le stock de dechets miniers 


\section{Conclusion}

Au cours de cette étude, les paramètres hydrodynamiques estimés et les conditions aux limites choisies permettent, à partir de l'application du modèle Modflow, de décrire le fonctionnement hydrodynamique du stock de déchets miniers de Carnoulès. L'écoulement se fait de l'amont vers la digue et est essentiellement guidé par le drain souterrain. Les débits à la sortie du stock de déchets miniers ont pu être reconstitués par le modèle. Ces débits ont donc permis d'estimer les flux de matières.

La durée de vie géochimique est supérieure à 100 ans. Il faudra donc atteindre une période correspondante pour voir atténuer naturellement cette pollution. Compte tenue du coût de la mise en place et de l'entretien sur cette longue période de vie du stock de déchets miniers, les techniques de traitement passif (biologique) et celles basées sur le blocage des réactions géochimiques seront les meilleures à explorer.

\section{Remerciements}

Ce travail a été financé par le programme «Européen Passive In situ Remediation Acide Mine Industrial Drainage (PIRAMID) ». Une reconnaissance particulière à $M$. Marc LEBLANC, Directeur de recherche au CNRS- Montpellier, qui a été le coordonnateur principal dudit programme.

\section{Références citées}

Alkaaby A., 1986. Conglomerats mineralisés (Pb$\mathrm{Ba}-\mathrm{Fe}$ ) du Trias basal sur la bordure Sud-Est des Cevennes: Exemple du système fluviatile en tresse de Carnoulès (Gard). Mémoire DEA. Université de Montpellier II, France. 154 pp.

Alkaaby A., Leblanc M. \& Perisol M., 1985. Minéralisation diagénétique précoce $(\mathrm{Pb}-\mathrm{Zn}-\mathrm{Ba})$ dans un environnement détritique continental : cas du trias de Carnoulès (Gard. France). C.R. Acad. Paris 300. pp 919-922.

Appia L. C., 1984. Le Trias Evaporitique du SE de la France : du bassin profond salifère à la bordure cévenole détritique et sulfatée (Largentière, Lodève). Thèse $3^{\text {éme }}$ Cycle. Université de Paris Sud, Centre Orsay, France. 613 pp.

Arthaud F. \& Matte P., 1975. Les décrochements tardi-hercyniens du SW de l'Europe, Géométrie et essai de reconstitution des conditions de déformation, Tectonophysique 25 : 139-217.

Bruneel O., Duran R., Koffi K., Fourcans A., ElbazPoulichet F., Leblanc M. \& Personne J-C., 2005. Microbial diversity and its relation to water chemistry in an As-rich tailings impoundment (Carnoulès, France). Geomicrobiol. J. 22 : 1-9.

Bozau E., Leblanc M., Seidel J. L. \& Stärk H. J., 2004. Light Rare Earth Elements enrichment in acidic mine Lake, Appl. Geochem. 19 : 261-271.

Casiot C., Morin M., Juillot F, Bruneel O., Personné J.C, Leblanc M. \& Duqesne K., 2001. Bacterial immobilization and oxidation of arsenic in acid mine drainage (Carnoulès creek, France), Water Res. 37 : 2929-2936.

Casiot C., Leblanc M., Bruneel O., Personné J.C, Koffi K. \& Elbaz-poulichet F., 2003. Geochemical processes controlling and formation of As-rich waters within a tailings impoundement (Carnoulès, France). Aquat. Geochem. 9 : 273-290.

Domenico A. P. \& Schwartz W. F., 1990. Physical and Chemical Hydrogeology. New York, USA : John Wiley and Sons. 824 pp.

Elbaz-Poulichet, F., Seyler, P., Maurice-Bourgoin, L., Guyot, J.L. \& Dupuy, C., 1999. Trace element geochemistry in the upper Amazon drainage basin (Bolivia). Chem. Geol. 157 : 319-334.

Hill M.C., 1994. Five computer programs for testing weighted residuals and calculating linear confidence and prediction intervals on results from the groundwater parameter-estimation computer program, MODFLOWP. US Geological Survey Open-File Report. 481 pp.

Koffi K., Leblanc m., Jourde H., Pistre S., Gouze P., Casiot C. \& Elbaz-Poulichet. F., 2003. Reverse oxidation zoning at a mine tailings stock generating arsenic-rich acid waters (Carnoulès, France). Mine Water Environ. J. 22 : 7-14.

Konikow, L.F., Goode, D.J. \& Hornberger, G.Z., 1996. A three-dimensional method-ofcharacteristics solute transport model (MOC3D). US Geological Survey Water Resources Investigations Report 96-4267. 87 p.

Leblanc M., Casiot C., Elbaz-poulichet F. \& Personné C., 2002. Arsenic removal by oxidizing bacteria in a heavily arsenic contaminated acid mine drainage system (Carnoulès, France). In : Younger P.L \& Robins NS. Eds. Mine water 
hydrogeology and geochemistry. Geological Society of London Special Publication. Kluwer Academic Publishers: Dordrecht. pp 215-239.

Leenhardt R., 1972. Le gîte plomb-zincifère de la croix de palières. Bull. B.R.G.M., Sect. II. 3 : 1-12.

Maree J. P., Hlabela P., Nongovhela R., Geldenhuys A. J., Mbhele N., Nevhulandzi T., \& Wanders F. B., 2004. Treatment of mine water for sulphate and metal removal using Barium Sulphide. Mine water Environ. J. 23 : $195-203$.

Morin G., Juillot F., Casiot C., Bruneel O., Personne J. C., Leblanc M., Ildefonse P. \& Cals G., 2003. Bacterial formation of Tooeleite and mixed ArsenicV-Iron III Gels in the Carnoulès Acid Drainage, France. A XANES, XRD, and SEM study. Environ. Sci. Technol. 37 : 1705-1712.

Nordstrom D., 1982. Aqueous pyrite oxidation and the consequent formation of secondary iron minerals. Soil Sci. Soc. Am. 10 : 37-62.
Seguret M. \& Proust F., 1965. L'évolution tectonique post hercynienne de la bordure mésozoïque des Cévennes Méridionales entre Alès et Ganges, Bull. Soc. Géol. Fr. 7 : 85-92.

Vialette Y. \& Sabourdy G., 1977. Granite de l'Aigoual dans le massif des Cévennes. Bull. Soc. Géol. Fr. 3 : 130-132.

Winston B., 1998. MODFLOW-related freeware and shareware resources on the internet. Comput. Geosci. 25 : 377-382.

Younger P.L., Banwart A.S. \& Hedin S.R., 2002. Mine Water: Hydrology, Pollution, Remediation. Environ. pollut. 5 : 42-46.

Zheng C., 1990. MT3D, A modular three dimensional transport model. S. S. Papadopoulos and associates, Bethesda, MD, In Winston R. B., MODFLOW related freeware and shareware resources on the internet. Comput. Geosci. 25 : 377-382. 